\title{
ICSP response to 'Science depends on nomenclature, but nomenclature is not science'
}

\author{
Iain C. Sutcliffe @D, David R. Arahal, Markus Göker and Aharon Oren
}

The Executive Board of the International Committee on Systematics of Prokaryotes (ICSP) read with much interest the recent comment by Lloyd and Tahon (Science depends on nomenclature, but nomenclature is not science. Nat. Rev. Microbiol. 20, 123-124 (2022) $)^{1}$. The authors raise various criticisms of recent ICSP activities to which we respond here.

Lloyd and Tahon ${ }^{1}$ highlight some consequences of the recent decision by the ICSP to revise the International Code of Nomenclature of Prokaryotes (ICNP) ${ }^{2}$ to recognize the taxonomic rank of phylum ${ }^{3}$. This decision, voted on by the ICSP in early 2021, redressed a historic oversight in that the rank of phylum was not included in earlier versions of the ICNP. This revision is important as, before then, any names for prokaryotic phyla were by definition colloquial and thus lacked standing in nomenclature (were not 'validly published names'). By contrast, phyla can now be given validly published names. As a result, Oren and Garrity validly published names for 42 phyla $^{4}$. However, following the new ICNP rules for how phylum names should be formed (specifically, taking a stem from the name of a contained genus, combined with the suffix -ota) means that some of the historic colloquial names (such as Firmicutes) cannot be adapted into readily recognizable (homophonic) validly published phylum names. Regrettably, in taking issue with some of the names proposed by Oren and Garrity ${ }^{4}$, Lloyd and Tahon ${ }^{1}$ muddle the action of these authors as independent individuals with the actions of the ICSP in revising the ICNP: amending the ICNP to permit the naming of phyla is one thing; proposing names for phyla in accordance with the Rules of the ICNP is completely separate. The ICSP recognizes that Oren and Garrity ${ }^{4}$ proposing replacements for some commonly used colloquial names may cause some short-term displeasure or misunderstanding but also emphasize that this will be offset by the clear long-term benefit to the research community of now being able to name prokaryotic phyla under the ICNP. Moreover, once names are proposed the community still decides which to adopt, although experience suggests that the scientific community will rapidly adjust. Similarly, how names are listed in databases is a matter of choice for their curators (although ICSP naturally encourages the use of correct names, as defined in the $\mathrm{ICNP}^{2}$ ).

In addition, Lloyd and Tahon ${ }^{1}$ take issue with the ICSP decision in 2020 (by 17 votes to 6) to reject proposals to accept DNA sequence as type ${ }^{5}$, thereby reinforcing the centrality of culture and deposit of type strains to the process of naming prokaryotes and, conversely, excluding the as-yet-uncultivated 'microbial dark matter' from being named under the ICNP. The reasons underpinning the ICSP commitment to publicly accessible type strains are a commitment to standardization and reproducibility, with type strains as the gold standard for use as a reference in future studies. It should be noted that the voting of the members of the ICSP took place after several years of discussion in various fora and a vigorous public email discussion, which has been minuted ${ }^{5}$. Furthermore, those working with uncultivated taxa do at least have the opportunity to provide them with placeholder Candidatus names, as described in Appendix 11 of the ICNP ${ }^{2,6}$. Although these names are not recognized within the Rules of the ICNP, and therefore lack standing in nomenclature, it is clear that they facilitate communication and, thus far, most names have been retained when Candidatus taxa have been cultured and their names validly published ${ }^{6}$. The ICSP agrees that it is important to promote the use of Candidatus names and to encourage stability in nomenclature (and respectful science) by retaining such names if the taxa are eventually cultured. Lloyd and Tahon also note that the ICNP rules regarding type strain accessibility present challenges for some researchers in countries where their governments may impose restrictions on sharing strains, as recently raised elsewhere ${ }^{7}$. The ICSP has already acknowledged this challenge and is willing to work with affected scientists ${ }^{8}$.

Finally, Lloyd and Tahon ${ }^{1}$ criticize the lack of diversity in the ICSP members. This is a valid criticism, albeit one that the ICSP was already alert to. The ICSP membership is primarily comprised of delegates from member societies of the International Union of Microbiological Societies (IUMS), as specified in our Statutes ${ }^{9}$. Ideally, ICSP would have better representation both from parts of the world that are currently under-represented and from a wider range of scientific 'end-user' communities, such as environmental microbiologists. Addressing this will likely require discussion with IUMS about how we can structure our membership and we commit to undertake this. Moreover, any interested party can propose emendments to the ICNP by following the process outlined in the ICSP Statutes?. We also emphasize that the ICSP is committed to openness through publication of its minutes, either in the International Journal of Systematic and Evolutionary Microbiology or on our website, and now conducts its discussions using electronic forums, including the Slack platform ${ }^{3,5,10}$.

Regrettably, the Comment by Lloyd and Tahon ${ }^{1}$ also contains extensive factual errors about the workings of the ICSP and concerning the relationship between classification and nomenclature. The ICSP only governs nomenclature, via maintaining the ICNP ${ }^{2,9}$, whereas classification is left to the scientific community. One example is "The ICSP relies on strict nomenclature rules and experts in microbial subgroups to ensure that nomenclature is stable and follows a polyphasic classification combining phenotype and genotype". In fact, the ICNP makes no comment on how classification should be performed and indeed Principle 1(4) of the $\mathrm{ICNP}^{2}$ very prominently protects freedom of 'taxonomic opinion', whilst the ICSP Statutes ${ }^{9}$ specify that the Subcommittees on Taxonomy ('subgroups') "cannot legislate on classification but may contribute materially towards the general acceptance of a classification". Decisions regarding appropriate methodological approaches (including the need for 'polyphasic classification') rest with editors (and peer reviewers) of journals publishing taxonomic studies. Multiple other errors are documented in a table that can be accessed on the ICSP website.

lain C. Sutcliffe (D) ${ }^{1 \times}$, David R. Arahal ${ }^{2}$, Markus Göker ${ }^{3}$ and Aharon Oren ${ }^{4}$ ${ }^{1}$ Northumbria University, Faculty of Health and Life Sciences, Newcastle Upon Tyne, UK. ${ }^{2}$ Departamento de Microbiología y Ecología Universitat de València, Valencia, Spain. ${ }^{3}$ Leibniz Institute DSMZ - German Collection of Microorganisms and Cell Cultures, Braunschweig, Germany. ${ }^{4}$ The Hebrew University of Jerusalem, The Institute of Life Sciences, Edmond J. Safra Campus - Givat Ram, Jerusalem, Israel.

凶e-mail: iain.sutcliffe@northumbria.ac.uk https://doi.org/10.1038/s41579-022-00706-z 


\section{CORRESPONDENCE}

1. Lloyd, K. G. \& Tahon, G. Science depends on nomenclature, but nomenclature is not science. Nat. Rev. Microbiol. 20, 123-124 (2022).

2. Parker, C. T., Tindall, B. J. \& Garrity, G. M. International Code of Nomenclature of Prokaryotes. Prokaryotic Code (2008 Revision). Int. J. Syst. Evol. Microbiol. 69, S1-S111 (2019).

3. Oren, A., Arahal, D. R., Rossellō-Móra, R., Sutcliffe, I. C. \& Moore, E. R. B. Emendation of Rules 5b, 8, 15 and 22 of the International Code of Nomenclature of Prokaryotes to include the rank of phylum. Int. J. Syst. Evol. Microbiol. 71 004851 (2021)

4. Oren, A. \& Garrity, G. M. Valid publication of the names of forty-two phyla of prokaryotes. Int. J. Syst. Evol. Microbiol. 71, 005056 (2021)

5. Sutcliffe, I. C., Dijkshoorn, L. \& Whitman, W. B. Minutes of the International Committee on Systematics of Prokaryotes online discussion on the proposed use of gene sequences as type for naming of prokaryotes, and outcome of vote. Int. J. Syst. Evol. Microbiol. 70, 4416-4417 (2020).

6. Pallen, M. J. The status Candidatus for uncultured taxa of Bacteria and Archaea: SWOT analysis. Int. J. Syst. Evol. Microbiol. 71, 005000 (2021).

7. Rahi, P. Regulating access can restrict participation in reporting new species and taxa. Nat. Microbiol. 6 , 1469-1470 (2021).

8. International Committee on Systematics of Prokaryotes. Position of the ICSP. ICSP https:// www.the-icsp.org/images/ICSP_Nagoya_position and_support.pdf (2022).

9. Whitman, W. B. et al. Request for revision of the Statutes of the International Committee on Systematics of Prokaryotes. Int. J. Syst. Evol. Microbiol. 69, 584-593 (2019).
10. Oren, A., Arahal, D. R., Rosselló-Môra, R., Sutcliffe, I. C. $\&$ Moore, E. R. B. Public discussion on a proposed revision of the International Code of Nomenclature of Prokaryotes. Int. J. Syst. Evol. Microbiol. 71, 004918 (2021).

\section{Acknowledgements}

The views and conclusions contained in this document are those of the authors and represent the views of the Executive Board of the International Committee on Systematics of Prokaryotes.

Competing interests

The authors declare no competing interests.

\section{RELATED LINKS}

ICSP website: https://www.the-icsp.org/publications 